Preprint typeset in JHEP style - HYPER VERSION

TIFR/TH/07-31

\title{
Kinematical Analogy for Marginal Dyon Decay
}

\author{
Anindya Mukherjee*, Sunil Mukhi ${ }^{\dagger}$, and Rahul Nigam ${ }^{\ddagger}$ \\ Tata Institute of Fundamental Research, \\ Homi Bhabha Rd, Mumbai 400 005, India
}

\begin{abstract}
We describe a kinematical analogy for the marginal decay of $\frac{1}{4}$-BPS dyons in 4-dimensional $\mathcal{N}=4$ string compactifications. In this analogy, the electric and magnetic charges play the role of spatial momenta, the BPS mass plays the role of energy, and $\frac{1}{2}$-BPS dyons correspond to massless particles. Using $S O(12,1)$ "Lorentz" invariance and standard kinematical formulae in particle physics, we provide simple derivations of the curves of marginal stability. We also show how these curves map into the momentum ellipsoid, and propose some applications of this analogy.
\end{abstract}

KEYWORDS: String theory.

\footnotetext{
${ }^{*}$ Email: anindya_m@theory.tifr.res.in

${ }^{\dagger}$ Email: mukhi@tifr.res.in

${ }^{\ddagger}$ Email: rahulnig@theory.tifr.res.in
} 


\section{Introduction and review}

Dyonic black holes in type IIB superstring compactifications to four dimensions on $K 3 \times T^{2}$ have been the subject of much interest in recent times. The counting formula of Ref. [1] for the microstates of these black holes has been re-derived in different ways [2, 3, 4 and subtle jumps in the spectrum have been accounted for by invoking dyon decay across curves of marginal stability[0, 6, 7, 8, 9, 10, 11].

Marginal stability is understood in terms of a formula that expresses the BPS mass of a dyonic state in terms of electric and magnetic charges as well as the moduli of the compactification. The BPS mass formula in turn descends from the supersymmetry algebra in 10 dimensions. For BPS states, only the right-moving momenta (the 6 part of $(6,22)$ ) are relevant, so one expects to see an $S O(6)$ symmetry. However, the phenomenon of interest to us, namely marginal decay of dyons, involves a larger symmetry. In addition to the 6 relevant electric charges there are now 6 magnetic charges. As we will see, the formulae relevant to dyon decay turn out to have an $S O(12,1)$ invariant structure that does not inherit from a symmetry of the underlying string theory. We will exploit this symmetry to recast the kinematics of dyon decay in terms of particles propagating in $12+1$ dimensions. This allows us to use standard formulae from particle kinematics to study marginal dyon decay.

The system we work with is the $\mathcal{N}=4$ supersymmetric background in 4 spacetime dimensions corresponding to type IIA/IIB string theory compactified on $K 3 \times T^{2}$ or the heterotic string on $T^{6}$, the two being related by duality. In what follows we will use the duality frame appropriate for type IIB. The resulting four-dimensional system has $28 U(1)$ gauge fields along with their electric-magnetic duals, therefore we can have dyons of electric and magnetic charge $\vec{Q}$ and $\vec{P}$, each one being a 28-vector. These dyons will be $\frac{1}{2}$-BPS if $\vec{Q}, \vec{P}$ are parallel, and $\frac{1}{4}$-BPS otherwise.

The BPS mass formula depends on the charges carried by the dyons as well as the values of the compactification moduli. These are encoded as follows. Define the matrix:

$$
L \equiv \operatorname{diag}\left(1^{6} ;(-1)^{22}\right)
$$

There are 132 moduli that can be assembled into a symmetric matrix $M$ which is orthogonal with respect to the $L$ metric:

$$
M^{T}=M, \quad M^{T} L M=L
$$


For purposes of the BPS mass formula, the relevant inner products for the charge vectors are:

$$
Q^{2} \equiv \vec{Q}^{T}(M+L) \vec{Q}, \quad P^{2} \equiv \vec{P}^{T}(M+L) \vec{P}, \quad P \cdot Q \equiv \vec{P}^{T}(M+L) \vec{Q}
$$

Let us also define the right-moving projections of the charge vectors, $\vec{Q}_{R}, \vec{P}_{R}$, by:

$$
Q_{R}^{2} \equiv \vec{Q}_{R}^{T} \vec{Q}_{R}=\vec{Q}^{T}(M+L) \vec{Q}=Q^{2}
$$

and similarly for the other inner products. In this way the moduli-dependent inner product Eq. (3) for the charge vectors is replaced by a standard moduli-independent product while the moduli-dependence is moved to the vectors themselves ${ }^{1}$. In what follows, we will not always write the suffix $R$, since the inner products are by definition the same whether we are dealing with the projected or unprojected vector.

The BPS mass formula for general $\frac{1}{4}$-BPS dyons is [12, [13, 6]:

$$
M^{\mathrm{BPS}}(\vec{Q}, \vec{P})^{2}=\frac{1}{\tau_{2}}(\vec{Q}-\bar{\tau} \vec{P}) \cdot(\vec{Q}-\tau \vec{P})+2 \sqrt{\Delta(\vec{Q}, \vec{P})}
$$

where

$$
\Delta(\vec{Q}, \vec{P}) \equiv Q^{2} P^{2}-(P \cdot Q)^{2}
$$

and $\tau$ is the modular parameter of the torus ${ }^{2}$.

\section{Kinematic Analogy}

The formula Eq. (5) has a striking analogy to the energy-momentum dispersion relation for a relativistic point particle, $E^{2}=\vec{p}^{2}+m^{2}$. In this analogy, the BPS mass plays the role of the energy of the particle, while the dyonic charges play the role of momenta:

$$
\vec{p}=\frac{1}{\sqrt{\tau_{2}}}(\vec{Q}-\tau \vec{P})
$$

Conservation of dyonic charge corresponds to momentum conservation in the kinematical system. For this, it is crucial that $\tau$ be complex (in fact it has a strictly positive imaginary part). Then conservation of the imaginary part of the momentum is equivalent to conservation of the dyon's magnetic charge, while the real part ensures conservation of electric charge.

\footnotetext{
${ }^{1}$ This refers to all moduli other than those of the 2-torus, which are always displayed explicitly and do not appear in the inner product.

${ }^{2}$ Our conventions are those in Ref.[6] and differ by a factor of $\frac{1}{\sqrt{\tau_{2}}}$ from those in Ref[1].
} 
To complete the analogy, note that the condition of marginal stability in a decay: $M^{\mathrm{BPS}}=M_{1}^{\mathrm{BPS}}+M_{2}^{\mathrm{BPS}}+\cdots$ corresponds to energy conservation. Finally, the mass of the analogue particle is $\sqrt{2}(\Delta(\vec{Q}, \vec{P}))^{\frac{1}{4}}$. A $\frac{1}{2}$-BPS dyon has its electric and magnetic charge vectors proportional, so $\Delta(\vec{Q}, \vec{P})=0$. It therefore corresponds to a massless particle.

Because the momentum vector defined in Eq. (]) is complex, some care must be taken in defining the inner product. As each of $\vec{Q}, \vec{P}$ is effectively 6-dimensional (recall that they are all projected using a moduli-dependent matrix, as in Eq. (⿴囗十)), one can convert the momenta to real vectors in a 12-dimensional space with Lorentz group $S O(12,1)$. As we have noted, these Lorentz symmetries are symmetries of the dispersion relation and the kinematics of the decay process, but not of the underlying string theory.

This point can be clarified by considering a general dyonic particle having a momentum $\vec{k}$ in the noncompact three-dimensional space. For such a particle the full dispersion relation is:

$$
\begin{aligned}
E^{2} & =\vec{k}^{2}+\left(M^{\mathrm{BPS}}\right)^{2} \\
& =\vec{k}^{2}+\frac{1}{\tau_{2}}(\vec{Q}-\bar{\tau} \vec{P}) \cdot(\vec{Q}-\tau \vec{P})+2 \sqrt{\Delta(\vec{Q}, \vec{P})}
\end{aligned}
$$

If we restrict our attention to electrically charged particles, $\vec{P}=0$, the above equation simplifies to:

$$
E^{2}=\vec{k}^{2}+\frac{1}{\tau_{2}} \vec{Q}^{2}
$$

which can be thought of as the dispersion relation for either a massive particle in $4 \mathrm{~d}$ or a massless particle in 10d. This is the expected situation for a Kaluza-Klein particle whose electric charges are momenta along the toroidal directions. The dependence on the modulus $\tau$, though present, is trivial in this case and can be removed by rescaling the metric appropriately. However, once we introduce magnetic charges as well then the dispersion relation is nontrivially $\tau$-dependent and also contains the $\Delta$ factor. Now if we put $\vec{k}=0$ then we have the kinematic analogy of interest in the present work.

To recover the curves of marginal stability, consider a general two-body decay of a particle with energy-momentum $(E, \vec{p})$ into a pair of particles of energy-momentum $\left(E_{1}, \vec{p}_{1}\right)$ and $\left(E_{2}, \vec{p}_{2}\right)$. There are two Lorentz frames that are useful: the rest frame of the initial particle and the lab frame where the initial particle has a given spatial 
momentum. By working in the rest frame, the Lorentz invariant $p \cdot p_{1}$ is easily shown to be:

$$
p \cdot p_{1}=\frac{1}{2}\left(m^{2}+m_{1}^{2}-m_{2}^{2}\right)
$$

The same Lorentz invariant in the lab frame can be written:

$$
p \cdot p_{1}=m_{1}^{2}+\sqrt{\vec{p}_{1}^{2}+m_{1}^{2}} \sqrt{\vec{p}_{2}^{2}+m_{2}^{2}}-\vec{p}_{1} \cdot \vec{p}_{2}
$$

Equating the two, we have:

$$
\sqrt{\vec{p}_{1}^{2}+m_{1}^{2}} \sqrt{\vec{p}_{2}^{2}+m_{2}^{2}}-\vec{p}_{1} \cdot \vec{p}_{2}=\frac{m^{2}-m_{1}^{2}-m_{2}^{2}}{2}
$$

To apply this to the decay of a $\frac{1}{4}$-BPS dyon, we make the substitution in Eq. (7) as well as:

$$
\vec{p}_{1}=\frac{1}{\sqrt{\tau_{2}}}\left(\vec{Q}_{1}-\tau \vec{P}_{1}\right), \quad \vec{p}_{2}=\frac{1}{\sqrt{\tau_{2}}}\left(\vec{Q}_{2}-\tau \vec{P}_{2}\right)
$$

to find:

$$
\begin{aligned}
\sqrt{\left|\vec{Q}_{1}-\tau \vec{P}_{1}\right|^{2}+2 \tau_{2} \sqrt{\Delta_{1}}} \sqrt{\left|\vec{Q}_{2}-\tau \vec{P}_{2}\right|^{2}+2 \tau_{2} \sqrt{\Delta_{2}}} & -\operatorname{Re}\left(\vec{Q}_{1}-\tau \vec{P}_{1}\right) \cdot\left(\vec{Q}_{2}-\bar{\tau} \vec{P}_{2}\right) \\
& =\tau_{2}\left(\sqrt{\Delta}-\sqrt{\Delta_{1}}-\sqrt{\Delta_{2}}\right)
\end{aligned}
$$

where $\Delta_{i}=\Delta\left(\vec{Q}_{i}, \vec{P}_{i}\right)$. For fixed charge vectors $\vec{Q}_{i}, \vec{P}_{i}$ and moduli matrix $M$ this is an equation for the torus modular parameter $\tau$. In other words, this is the general curve of marginal stability!

We can relate this to previously derived forms of the curve, as in [6, 10, 11]. First let us consider the case of decay of a primitive $(\operatorname{gcd}(\vec{Q} \wedge \vec{P})=1) \frac{1}{4}$-BPS dyon into two $\frac{1}{2}$-BPS dyons. In this case the product particles are massless. Moreover, the charge vectors of the decay products are given by [6]:

$$
\begin{array}{ll}
\vec{Q}_{1}=a \vec{N}_{1}, & \vec{P}_{1}=c \vec{N}_{1} \\
\vec{Q}_{2}=b \vec{N}_{2}, & \vec{P}_{2}=d \vec{N}_{2}
\end{array}
$$

where

$$
\vec{N}_{1}=d \vec{Q}-b \vec{P}, \quad \vec{N}_{2}=-c \vec{Q}+a \vec{P}
$$

nd $a d-b c=1$. Thus the equation reduces to:

$$
|a-c \tau||b-d \bar{\tau}| \frac{\left|\vec{N}_{1}\right|\left|\vec{N}_{2}\right|}{\sqrt{\Delta}}-\operatorname{Re}\{(a-c \tau)(b-d \bar{\tau})\} \frac{\vec{N}_{1} \cdot \vec{N}_{2}}{\sqrt{\Delta}}=\tau_{2}
$$


Let

$$
\mathcal{E}=-\frac{\vec{N}_{1} \cdot \vec{N}_{2}}{\sqrt{\Delta\left(\vec{N}_{1}, \vec{N}_{2}\right)}}
$$

It is easily checked that

$$
\sqrt{1+\mathcal{E}^{2}}=\frac{\left|\vec{N}_{1}\right|\left|\vec{N}_{2}\right|}{\sqrt{\Delta\left(\vec{N}_{1}, \vec{N}_{2}\right)}}
$$

Using the above equations and the fact that $\Delta\left(\vec{N}_{1}, \vec{N}_{2}\right)=\Delta(\vec{Q}, \vec{P})$, the curve becomes:

$$
|a-c \tau||b-d \bar{\tau}| \sqrt{1+\mathcal{E}^{2}}+\operatorname{Re}\{(a-c \tau)(b-d \bar{\tau})\} \mathcal{E}=\tau_{2}
$$

Now one can take the second term to the right side and square both sides, whereupon the resulting quartic (in $\tau$ ) re-factorises into a perfect square of the familiar Sen circle equation. However there is a neater way to proceed. The above equation is the same as:

$$
|(a-c \tau)(b-d \bar{\tau})(\mathcal{E}+i)|+\operatorname{Re}(a-c \tau)(b-d \bar{\tau})(\mathcal{E}+i)=0
$$

which is equivalent to the two conditions:

$$
\begin{aligned}
& \operatorname{Im}(a-c \tau)(b-d \bar{\tau})(\mathcal{E}+i)=0 \\
& \operatorname{Re}(a-c \tau)(b-d \bar{\tau})(\mathcal{E}+i)<0
\end{aligned}
$$

The first of these is directly the equation of the Sen circle:

$$
\left(\tau_{1}-\frac{a d+b c}{2 c d}\right)^{2}+\left(\tau_{2}+\frac{\mathcal{E}}{2 c d}\right)^{2}=\frac{1}{4 c^{2} d^{2}}\left(1+\mathcal{E}^{2}\right)
$$

while the second one restricts us to the $\tau_{2}>0$ region of that circle.

Next consider decays into two $\frac{1}{4}$-BPS dyons, or one $\frac{1}{4}$-BPS and one $\frac{1}{2}$-BPS dyon. In this case the charges of the final states are parametrised as:

$$
\begin{array}{cl}
\vec{Q}_{1}=m_{1} \vec{Q}+r_{1} \vec{P}, & \vec{P}_{1}=s_{1} \vec{Q}+n_{1} \vec{P} \\
\vec{Q}_{2}=\left(m-m_{1}\right) \vec{Q}-r_{1} \vec{P}, & \vec{P}_{2}=-s_{1} \vec{Q}+\left(n-n_{1}\right) \vec{P}
\end{array}
$$

where the charges of the initial dyon are now $(m \vec{Q}, n \vec{P})$ with $\operatorname{gcd}(\vec{Q} \wedge \vec{P})=1$.

Substituting the above into Eq. (14), transposing the second term to the right hand side and squaring, one finds:

$$
\left(\tau_{1}-\frac{m \wedge n}{2 n s_{1}}\right)^{2}+\left(\tau_{2}+\frac{\mathcal{E}}{2 n s_{1}}\right)^{2}=\frac{1}{4 n^{2} s_{1}^{2}}\left((m \wedge n)^{2}+4 m n r_{1} s_{1}+\mathcal{E}^{2}\right)
$$


where

$$
\mathcal{E} \equiv-\frac{\vec{Q}_{1} \cdot \vec{P}_{2}-\vec{Q}_{2} \cdot \vec{P}_{1}}{\sqrt{\Delta}}=\frac{1}{\sqrt{\Delta}}\left(m s_{1} Q^{2}-n r_{1} P^{2}-(m \wedge n) Q \cdot P\right)
$$

and $m \wedge n=m_{1} n_{2}-m_{2} n_{1}$. This is the general curve of marginal stability found in [11].

\section{Momentum ellipsoid}

For two-body decay of an unstable particle in the lab frame, the final-state particle momenta are constrained to lie on an ellipsoid of revolution, obtained by rotating an ellipse in the forward and transverse momenta $p_{1, \|}$ and $p_{1, \perp}$ along the azimuthal angles around the beam axis. It is tempting to guess that the curve of marginal stability coincides with this ellipse, We will see below that this is roughly true but the relationship is more complicated than one might have expected.

Indeed this approach, in which $p$ and $p_{1}$ are treated as the independent variables, is not the easiest way to derive the curves of marginal stability, which in fact we have already done in the previous section by treating the final-state momenta $p_{1}, p_{2}$ as the independent variables. Nevertheless it is of some conceptual interest to understand how the curves of marginal stability are related to the momentum ellipsoid. One reason why the embedding we will obtain is rather complicated is that the momentum of the initial particle (which determines one axis of the ellipse) itself depends on the modular parameter $\tau$.

To find the momentum ellipsoid, we first eliminate $\vec{p}_{2}$ in Eq. (12) in favour of $\vec{p}, \vec{p}_{1}$. Then, taking the second term to the right hand side and squaring, we end up with the equation:

$$
m^{2}\left(p_{1, \|}-\frac{|\vec{p}|\left(m^{2}-m_{1}^{2}-m_{2}^{2}\right)}{2 m^{2}}\right)^{2}+\left(\vec{p}^{2}+m^{2}\right) p_{1, \perp}^{2}=\frac{1}{4} \lambda\left(m^{2}, m_{1}^{2}, m_{2}^{2}\right)\left(1+\frac{\vec{p}^{2}}{m^{2}}\right)
$$

where

$$
\lambda(a, b, c) \equiv a^{2}+b^{2}+c^{2}-2 a b-2 b c-2 c a
$$

Here $p_{1, \|}$ and $p_{1, \perp}$ are the components of $\vec{p}_{1}$ along and transverse to the beam. The momentum ellipsoid is the ellipse in Eq. (27) rotated about the beam axis.

For decays into two $\frac{1}{2}$-BPS states, we put $m_{1}=m_{2}=0$ and the momentum ellipsoid simplifies considerably into:

$$
\frac{\left(p_{1, \|}-\frac{|\vec{p}|}{2}\right)^{2}}{\frac{1}{4}\left(\vec{p}^{2}+m^{2}\right)}+\frac{p_{1, \perp}^{2}}{\frac{1}{4} m^{2}}=1
$$


Evidently the major axis of the ellipse is proportional to $\sqrt{\vec{p}^{2}+m^{2}}$, which from Eq. (đ) is $\tau$-dependent. Therefore the momentum ellipsoid itself varies with $\tau$, and the curve of marginal stability is technically not a subspace of a particular ellipsoid. This can be remedied by defining a new variable $\tilde{p}_{1}$ via:

$$
\tilde{p}_{1} \equiv \frac{p_{1, \|}-\frac{|\vec{p}|}{2}}{\sqrt{1+\frac{\vec{p}^{2}}{m^{2}}}}
$$

in terms of which the ellipse becomes a circle with a $\tau$-independent radius:

$$
\tilde{p}_{1}^{2}+p_{1, \perp}^{2}=\frac{1}{4} m^{2}
$$

From Eqs.(7),(13) we find:

$$
\begin{aligned}
p_{1, \|} & =\frac{1}{\sqrt{\tau_{2}}} \frac{\operatorname{Re}\left((\vec{Q}-\tau \vec{P}) \cdot\left(\vec{Q}_{1}-\bar{\tau} \vec{P}_{1}\right)\right)}{|\vec{Q}-\tau \vec{P}|} \\
p_{1, \perp} & =\frac{1}{\sqrt{\tau_{2}}} \frac{1}{|\vec{Q}-\tau \vec{P}|} \sqrt{|\vec{Q}-\tau \vec{P}|^{2}\left|\vec{Q}_{1}-\tau \vec{P}_{1}\right|^{2}-\left(\operatorname{Re}(\vec{Q}-\tau \vec{P}) \cdot\left(\vec{Q}_{1}-\bar{\tau} \vec{P}_{1}\right)\right)^{2}}
\end{aligned}
$$

from which one obtains:

$$
\begin{aligned}
\tilde{p}_{1} & =\frac{1}{\sqrt{\tau_{2}}} \frac{1}{|\vec{Q}-\tau \vec{P}|} \frac{\left(a d+b c-2 c d \tau_{1}\right) Q^{2}+\left(2 a b \tau_{1}-(a d+b c)|\tau|^{2}\right) P^{2}+\left(-2 a b+2 c d|\tau|^{2}\right) Q \cdot P}{\sqrt{\frac{1}{\tau_{2}}|\vec{Q}-\tau \vec{P}|^{2}+2 \sqrt{\Delta(\vec{Q}, \vec{P})}}} \\
p_{1, \perp} & =\frac{1}{\sqrt{\tau_{2}}} \frac{1}{|\vec{Q}-\tau \vec{P}|} \sqrt{\Delta(\vec{Q}, \vec{P})} \sqrt{(\operatorname{Re}(a-c \tau)(b-d \bar{\tau}))^{2}+\left(\mathcal{E}^{2}+1\right) \tau_{2}^{2}}
\end{aligned}
$$

where $\mathcal{E}$ has been defined in Eq. (18). As promised, this is rather complicated. One might hope to find a better parametrisation that leads to a simpler embedding.

\section{Multiparticle decays and the issue of codimension}

In order for the dyons in the final state to be BPS relative to each other, $\vec{Q}_{i, R}, \vec{P}_{i, R}$ must lie in the same plane as $\vec{Q}_{R}, \vec{P}_{R}{ }^{3}$. This condition implies that all decays other than two-body decays into $\frac{1}{2}$-BPS particles occur on codimension $\geq 2$ subspaces of moduli space, as noted in Refs. 10, 11.

We can incorporate this property into the kinematical analogy. Let us impose on our 12-dimensional space the structure of a linear symplectic manifold. Thus there is

\footnotetext{
${ }^{3}$ This condition also follows from the kinematics[10], we thank the referee for emphasizing this fact to us.
} 
a polarisation - a closed, non-degenerate 2-form that divides the space into 6 electric and 6 magnetic directions, each one a Lagrangian subspace analogous to coordinates and momenta in a phase space.

Consider a particle of arbitrary 12-momentum. We use the polarisation to divide this momentum into its electric and magnetic parts, each being a 6 -vector. Now consider the "diagonal" 6-manifold obtained by identifying the electric and magnetic subspaces. In this 6-manifold, the linear span of the electric and magnetic parts of the 12-momentum defines a plane (if these 6-vectors are non-parallel) or a line (if they are parallel). The former case is $\frac{1}{4}$-BPS while the latter is $\frac{1}{2}$-BPS.

In a two-body decay, if the original particle defines a plane in the "diagonal" space while the final particles define lines, then by momentum conservation the BPS condition is automatically satisfied. If the final state particles are $\frac{1}{2}$-BPS but three or more in number, or if at least one of them is $\frac{1}{4}$-BPS, then the dimensionality of the subspace of the diagonal space spanned is at least three. In this case, additional conditions on the moduli besides the marginal stability condition are required to make the decay possible 10, 11]. The embedding of codimension $\geq 2$ curves in the full moduli space has, however, not yet been given a precise description.

\section{Decay widths on marginal stability curves}

The kinematical analogy suggests that one consider decay and scattering processes involving dyons on curves of marginal stability. As a simple example, consider an ensemble of $\frac{1}{4}$-BPS dyons of a given charge, at a curve of marginal stability for decay into two $\frac{1}{2}$-BPS dyons. The ensemble will decay with a width given by a formula analogous to the classic formula for decay of a particle of mass $m$ into two identical massless particles, which in three noncompact space dimensions and in the rest frame of the decaying particle is (see for example Ref. [14]):

$$
\Gamma=\frac{1}{32 \pi \hbar m}|\mathcal{M}|^{2}
$$

where $\mathcal{M}$ is the matrix element for the process.

To apply this formula to the present case, the kinematics needs to be re-done in the lab frame of the decaying particle, and in 12 dimensions. Also we need to incorporate the true spacetime momenta of the initial and final state particles - the above expression should be thought of only as the factor in the decay width coming from the "analogue" momenta. We also need to take into account the quantisation rule for the "analogue" $12 \mathrm{~d}$ momenta, inherited from the quantisation of the original 
electric and magnetic charges. Finally, the matrix element $\mathcal{M}$ needs to be computed. It seems quite plausible that everything here is computable in string theory. One can similarly consider scattering cross-sections for dyons.

\section{Conclusions}

We have presented an analogy that maps the marginal stability conditions for $\frac{1}{4}$ BPS dyons into energy-momentum conservation in an analogue particle problem

in $12+1$ dimensions. $\frac{1}{4}$-BPS states behave like massive particles and $\frac{1}{2}$-BPS states like massless particles. The analogy provides a simple way to understand curves of marginal stability and may be useful both in deriving these curves in other situations and in suggesting ways to think about physical processes involving $\frac{1}{4}$-BPS dyons.

\section{Acknowledgements}

We would like to thank Rajesh Gopakumar and Sandip Trivedi, as well as all the participants of the Indian Strings Meeting, HRI Allahabad, October 2007, for useful discussions. The work of AM was supported in part by CSIR Award No. 9/9/256(SPM$5) / 2 \mathrm{~K} 2 /$ EMR-I. As always, the generous support of the people of India is gratefully acknowledged.

\section{References}

[1] R. Dijkgraaf, E. P. Verlinde, and H. L. Verlinde, Counting dyons in $N=4$ string theory, Nucl. Phys. B484 (1997) 543-561, hep-th/9607026.

[2] D. Shih, A. Strominger, and X. Yin, Recounting dyons in $N=4$ string theory, JHEP 10 (2006) 087, hep-th/0505094.

[3] D. Gaiotto, Re-recounting dyons in $N=4$ string theory, hep-th/0506249.

[4] J. R. David and A. Sen, CHL dyons and statistical entropy function from D1-D5 system, JHEP 11 (2006) 072, hep-th/0605210.

[5] A. Dabholkar and D. Gaiotto, Spectrum of CHL dyons from genus-two partition function, hep-th/0612011.

[6] A. Sen, Walls of marginal stability and dyon spectrum in $n=4$ supersymmetric string theories, JHEP 05 (2007) 039, hep-th/0702141.

[7] A. Dabholkar, D. Gaiotto, and S. Nampuri, Comments on the spectrum of CHL dyons, hep-th/0702150. 
[8] A. Sen, Two centered black holes and N=4 dyon spectrum, arXiv:0705.3874.

[9] M. C. N. Cheng and E. Verlinde, Dying dyons don't count, JHEP 09 (2007) 070, arXiv:0706.2363.

[10] A. Sen, Rare decay modes of quarter BPS dyons, arXiv:0707.1563.

[11] A. Mukherjee, S. Mukhi, and R. Nigam, Dyon death eaters, JHEP 10 (2007) 037, arXiv:0707.3035.

[12] M. Cvetic and D. Youm, Dyonic BPS saturated black holes of heterotic string on a six torus, Phys. Rev. D53 (1996) 584-588, hep-th/9507090.

[13] M. J. Duff, J. T. Liu, and J. Rahmfeld, Four-dimensional string-string-string triality, Nucl. Phys. B459 (1996) 125-159, hep-th/9508094.

[14] D. J. Griffiths, Introduction to elementary particles. Wiley, New York, 1987. 